\title{
Self-identification method of arrangement and effective pressure areas for a vibration-isolation table supported with a redundant number of pneumatic actuators
}

\author{
Yohei HOSHINO*, Soichiro SUZUKI ${ }^{*}$, Kenji TAKAGI ${ }^{* *}$ and Yukinori KOBAYASHI ${ }^{* * *}$ \\ * Faculty of Engineering, Kitami Institute of Technology \\ 165 Koen-cho, Kitami-shi, Hokkaido, 090-8507, Japan \\ E-mail: hoshinoy@mail.kitami-it.ac.jp \\ ** Department of Mechanical Engineering, Kitami Institute of Technology \\ 165 Koen-cho, Kitami-shi, Hokkaido, 090-8507, Japan \\ *** Faculty of Engineering, Hokkaido University \\ N13W8, Kita-ku, Sapporo, Hokkaido, 060-8628, Japan
}

Received 24 December 2014

\begin{abstract}
For the purpose of developing a vibration-isolation table with flexibility and/or scalability (which means "with adaptability to desired load specification"), this study investigates a self-identification method with regard to positions or arrangement and effective pressure areas of pneumatic actuators which support a vibration-isolation table. The proposed identification method is a fundamental technique necessary to construct a scalable vibration-isolation table in which the actuators supporting the table can be added or removed flexibly according to the load demand, easily, and in a short period of time. First, this study constructs a mathematical model for a vibration-isolation table supported by a redundant number of actuators and a type 1 digital servo controller to control the position and inclination angles of the table. Next, we derive the identification method for obtaining the position of the added pneumatic actuator and the effective pressure areas of the pneumatic actuators. We then demonstrate the proposed identification method by numerical simulations using our detailed mathematical model. Finally, this study shows an example of the application of the proposed identification method coupled with a force redistribution technique by addition of an actuator into the vibration-isolation table system during continuous operation. The validation and success of our method was confirmed by the displacement and pressure responses of the vibration-isolation table.
\end{abstract}

Key words : Vibration-isolation table, Pneumatic actuator, Redundant actuator, Scalability, Identification

\section{Introduction}

Vibration-isolation tables have been widely used to support semiconductor exposure apparatuses among other applications, and are used in the prevention of transmitted vibrations from mechanical devices mounted on the apparatuses themselves as well as through the ground. For example, the vibration-isolation is necessary not only to protect the apparatuses from the damage by strong vibration due to earthquakes, but also to prevent the semiconductor apparatuses from any vibrations through the exposure-processes, because the vibrations directly deteriorate the processing resolution and throughput in photo-lithography used in the semiconductor exposure apparatuses. Much research has been devoted to active vibration control for vibration-isolation tables and is further being investigated to achieve higher vibration-isolation capability by many researchers including Erin, et al. (1998), Kim, et al. (2001), Zuo, et al. (2005), Hashimoto, et al. (2007), Hoshino, et al. (2007, 2009, 2010), Shirani and Wakui (2010), Shin, et al. (2010), and Chen and Shih (2010), to name a few.

In recent years, product development cycles have become shorter in the manufacturing industries. Lowering energy consumption in manufacturing is required for reducing not only the manufacturing cost but also environmental load. 
Therefore, a more flexible production process, which can be reconstructed easily according to fast changes in market demands, is required for the future of manufacturing. Since semiconductor production apparatuses have become bigger due to the high demand for large-scale devices such as liquid crystal displays (LCDs), large-scale vibration-isolation tables with better vibration-isolation performance are necessary to support and avoid vibrations from these apparatuses. Therefore, the aforementioned flexible production process must strongly adhere to semiconductor production apparatuses that ensure reduction in both manufacturing cost and energy consumption.

For the purpose of developing such a scalable vibration-isolation table with adaptability to the desired load specification, this study investigates a self-identification method of positions and effective pressure areas of a redundant number of pneumatic actuators which support the vibration-isolation table. The proposed identification method gives a fundamental technique necessary to construct a scalable vibration-isolation table which can increase or decrease the number of actuators supporting the table flexibly, easily, and in a short period of time. This study demonstrates the proposed method by numerical simulations using the detailed model of the vibration-isolation table supported with a redundant number of pneumatic actuators.

\section{Nomenclature}

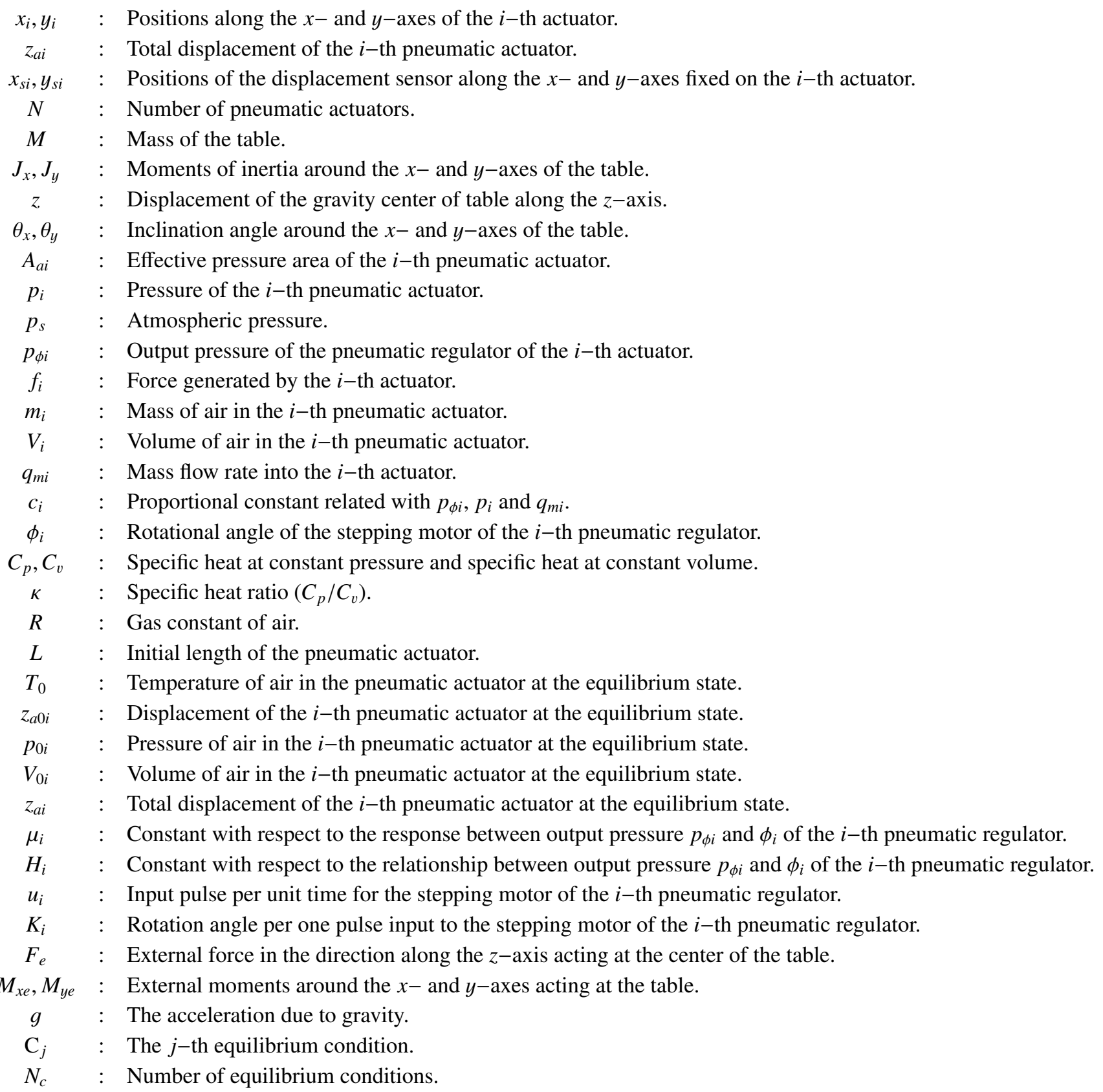




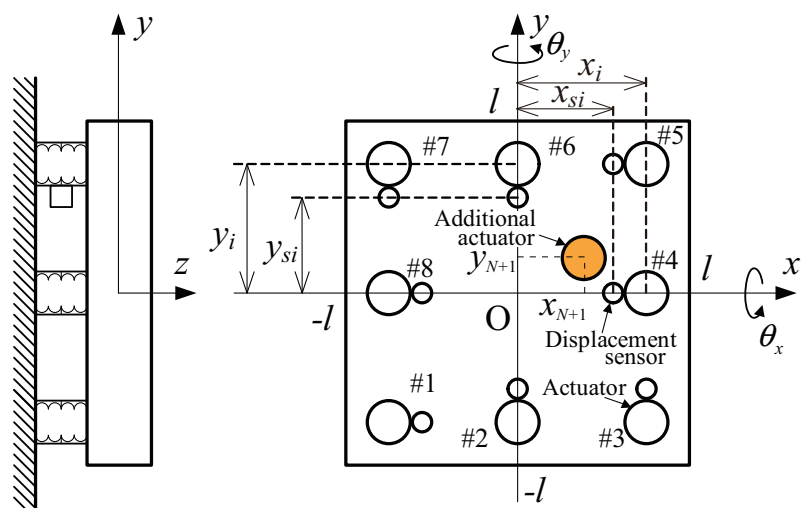

Fig. 1 3DOF model of the vibration-isolation table supported with a redundant number of actuators.

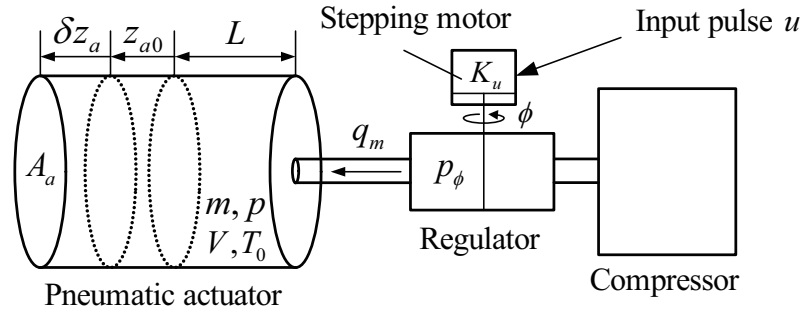

Fig. 2 Pneumatic actuator controlled by a pneumatic regulator driven by a stepping motor.

(o) : Differentiation operator with respect to time for the symbol (o).

$\Delta(\circ)$ : Backward difference operator for the symbol (o).

$\Delta t \quad$ : Sampling period of the controller.

(o) : Symbol which indicates identified values for the symbol (o).

$k \quad$ : Number of sample steps of the controller.

\section{Mathematical model and controller design}

\subsection{Mathematical model for the vibration-isolation table}

This study derives a mathematical model for a vibration-isolation table supported with a redundant number of actuators as shown in Fig. 1 (Hoshino et al., 2012, 2013, Maeda et al., 2013). The coordinate system O-xyz is defined as an orthogonal coordinate system. The $z$-axis passes through the center of gravity of the table in the equilibrium state, and $z$ represents the vertical displacement of the table. The inclination angles of the table around the $x-$ and $y$-axes passing through the center of gravity of the table are represented by $\theta_{x}$ and $\theta_{y}$, respectively. This study considers the 3 degrees of freedom (DOF) of the table's motion. The table is supported by a redundant number of pneumatic actuators, which are driven by stepping motors as shown in Fig. 2. The equation of motion for the table is expressed as

$$
\boldsymbol{M} \ddot{\boldsymbol{X}}=\boldsymbol{G}_{1} \boldsymbol{A}_{a}\left(\boldsymbol{p}-\boldsymbol{p}_{s}\right)-\boldsymbol{M g}+\boldsymbol{F}_{e},
$$

where

$$
\begin{aligned}
\boldsymbol{M} & =\operatorname{diag}\left[\begin{array}{lll}
M & J_{x} & J_{y}
\end{array}\right], \boldsymbol{g}=\left[\begin{array}{lll}
g & 0 & 0
\end{array}\right]^{T}, \quad \boldsymbol{A}_{a}=\operatorname{diag}\left[\begin{array}{lll}
A_{a 1} & \cdots & A_{a N}
\end{array}\right] \\
\boldsymbol{G}_{1} & =\left[\begin{array}{ccc}
1 & \cdots & 1 \\
y_{1} & \cdots & y_{N} \\
-x_{1} & \cdots & -x_{N}
\end{array}\right], \boldsymbol{X}=\left[\begin{array}{c}
z \\
\theta_{x} \\
\theta_{y}
\end{array}\right], \quad \boldsymbol{p}=\left[\begin{array}{c}
p_{1} \\
\vdots \\
p_{N}
\end{array}\right], \quad \boldsymbol{p}_{s}=\left[\begin{array}{c}
p_{s} \\
\vdots \\
p_{s}
\end{array}\right]_{N \times 1}, \boldsymbol{F}_{e}=\left[\begin{array}{c}
F_{e} \\
M_{x e} \\
M_{y e}
\end{array}\right]_{N \times 1} .
\end{aligned}
$$

Here, the mass of the table is represented by $M$, and the moments of inertia about the $x$ - and $y$-axes are represented by $J_{x}$ and $J_{y}$, respectively. The effective pressure area and the pressure of air of the $i$-th actuator are represented by $A_{a i}$ and $p_{i}$, respectively. The acceleration due to gravity and the atmospheric pressure are represented by $g$ and $p_{s}$, respectively. The matrix $\boldsymbol{G}_{1}$ is constructed from the positions of the actuators. In addition, the external force acting on the table is represented by $F_{e}$, and the external moments about the $x$ - and $y$-axes acting on the table are represented by $M_{x e}$ and $M_{y e}$. The number of actuators is represented by $N$ and the relationship between the pressure $p_{i}$ and the total displacement $z_{a i}$ of the $i$-th pneumatic actuator is expressed as follows (Erin, et al., 1998):

$$
\dot{p}_{i}=-\kappa \frac{p_{0 i} A_{a i}}{V_{0 i}} \dot{z}_{a i}-\kappa \frac{R T_{0} c_{i}}{V_{0 i}} p_{i}+\kappa \frac{R T_{0} c_{i}}{V_{0 i}} p_{\phi i}
$$

where $\kappa=C_{p} / C_{v}$ represents specific heat ratio determined by the specific heat at constant volume, $C_{v}$, and the specific heat at constant pressure, $C_{p}$. The gas constant is represented by $R$, and $c_{i}$ represents the proportionality constant related with the pressure $p_{i}$, the output pressure of pneumatic regulator $p_{\phi i}$, and the mass flow rate $q_{m i}$ of the $i$-th actuator. There 
are short time delays in the responses from the rotations of the stepping motors $\phi_{i}$ to the output pressures of the pneumatic regulators $p_{\phi i}$. The relation between $\phi_{i}$ and $p_{\phi i}$ is expressed as a first order delay system,

$$
\dot{p}_{\phi i}=\mu_{i}\left(H_{i} \phi_{i}-p_{\phi i}\right)
$$

The rotation angles of the stepping motors that control the output pressures of the regulators are expressed as

$$
\dot{\phi}_{i}=K_{u} u_{i}
$$

where $K_{u}$ represents the proportionality constants. Also, $z_{a i}$ in Eq. (2) satisfies

$$
\left[\begin{array}{lllll}
\dot{z}_{a 1} & \cdots & \dot{z}_{a i} & \cdots & \dot{z}_{a N}
\end{array}\right]^{T}=\boldsymbol{G}_{1}^{T} \dot{\boldsymbol{X}}
$$

from the geometric relationship of the position of the actuators. From Eqs. (1)-(5), the state equations for the vibrationisolation table are expressed as

$$
\begin{aligned}
& \dot{\boldsymbol{x}}=\boldsymbol{A} \boldsymbol{x}+\boldsymbol{B} \boldsymbol{\phi}+\boldsymbol{D} \boldsymbol{p}_{s}+\boldsymbol{E}_{g} \boldsymbol{g}+\boldsymbol{E}_{e} \boldsymbol{F}_{e}, \\
& \dot{\boldsymbol{\phi}}=\boldsymbol{K}_{u} \boldsymbol{u}
\end{aligned}
$$

where

$$
\begin{aligned}
& \boldsymbol{A}=\left[\begin{array}{cc|cc}
\mathbf{0} & \mathbf{I} & \mathbf{0} & \mathbf{0} \\
\mathbf{0} & \mathbf{0} & \boldsymbol{M}^{-1} \boldsymbol{G}_{1} \boldsymbol{A}_{a} & \mathbf{0} \\
\hline \mathbf{0} & \boldsymbol{W}_{1} \boldsymbol{G}_{1}^{T} & -\boldsymbol{W}_{2} & \boldsymbol{W}_{2} \\
\mathbf{0} & \mathbf{0} & \mathbf{0} & -\boldsymbol{\mu}
\end{array}\right], \boldsymbol{B}=\left[\begin{array}{c}
\mathbf{0} \\
\mathbf{0} \\
\hline \mathbf{0} \\
\boldsymbol{\mu} \boldsymbol{H}
\end{array}\right], \boldsymbol{D}=\left[\begin{array}{c}
\mathbf{0} \\
-\boldsymbol{M}^{-1} \boldsymbol{G}_{1} \boldsymbol{A}_{a} \\
\mathbf{0} \\
\mathbf{0}
\end{array}\right], \boldsymbol{E}_{\boldsymbol{g}}=\left[\begin{array}{c}
\mathbf{0} \\
-\mathbf{I}_{3 \times 3} \\
\hline \mathbf{0} \\
\mathbf{0}
\end{array}\right], \boldsymbol{E}_{\boldsymbol{e}}=\left[\begin{array}{c}
\mathbf{0} \\
\frac{\boldsymbol{M}^{-1}}{\mathbf{0}} \\
\mathbf{0}
\end{array}\right], \\
& \boldsymbol{x}=\left[\begin{array}{lllll}
\boldsymbol{X}^{T} & \dot{\boldsymbol{X}}^{T} & \mid \boldsymbol{p}^{T} & \boldsymbol{p}_{\phi}^{T}
\end{array}\right]^{T}, \boldsymbol{u}=\left[\begin{array}{lll}
u_{1} & \cdots & u_{N}
\end{array}\right]^{T}, \boldsymbol{\phi}=\left[\begin{array}{lll}
\phi_{1} & \cdots & \phi_{N}
\end{array}\right]^{T}, \quad \boldsymbol{p}_{\phi}=\left[\begin{array}{lll}
p_{\phi 1} & \cdots & p_{\phi N}
\end{array}\right]^{T}, \\
& \boldsymbol{\mu}=\operatorname{diag}\left[\begin{array}{lll}
\mu_{1} & \cdots & \mu_{N}
\end{array}\right], \quad \boldsymbol{H}=\operatorname{diag}\left[\begin{array}{llll}
H_{1} & \cdots & H_{N}
\end{array}\right], \quad \boldsymbol{K}_{u}=\operatorname{diag}\left[\begin{array}{llll}
K_{u} & \cdots & K_{u}
\end{array}\right]_{N \times N}, \\
& \boldsymbol{W}_{1}=\operatorname{diag}\left[\begin{array}{lll}
-\frac{\kappa p_{01} A_{a 1}}{V_{01}} & \cdots & -\frac{\kappa p_{0 N} A_{a N}}{V_{0 N}}
\end{array}\right], \boldsymbol{W}_{2}=\operatorname{diag}\left[\begin{array}{lll}
\frac{\kappa R T_{0} c_{1}}{V_{01}} & \cdots & \frac{\kappa R T_{0} c_{N}}{V_{0 N}}
\end{array}\right] .
\end{aligned}
$$

\subsection{Controller for position and inclination angle of the vibration-isolation table}

As an example, this study utilizes a type 1 digital optimal servo controller to control and stabilize the position and inclination angle of the vibration-isolation table (Hoshino et al., 2012, 2013, Maeda et al., 2013). To construct the type 1 digital servo controller effectively using the integral characteristics of the stepping motor given by Eq. (7), the discretetime state equation of state equation (6) is reconstructed as

$$
\boldsymbol{x}(k+1)=\boldsymbol{A}_{d} \boldsymbol{x}(k)+\boldsymbol{B}_{d} \boldsymbol{\phi}(k)+\boldsymbol{D}_{d} \boldsymbol{p}_{s}+\boldsymbol{E}_{g d} \boldsymbol{g}+\boldsymbol{E}_{e d} \boldsymbol{F}_{e},
$$

where

$$
\boldsymbol{y}(k)=\boldsymbol{C}_{d} \boldsymbol{x}(k)=\left[z(k) \theta_{x}(k) \theta_{y}(k)\right]^{T},
$$

and $\boldsymbol{A}_{d}=e^{\boldsymbol{A} \Delta t}, \boldsymbol{B}_{d}=\left(\int_{0}^{\Delta t} e^{\boldsymbol{A} \tau} d \tau\right) \boldsymbol{B}, \boldsymbol{C}_{d}=\boldsymbol{C}, \boldsymbol{D}_{d}=\left(\int_{0}^{\Delta t} e^{\boldsymbol{A} \tau} d \tau\right) \boldsymbol{D}, \boldsymbol{E}_{g d}=\left(\int_{0}^{\Delta t} e^{\boldsymbol{A} \tau} d \tau\right) \boldsymbol{E}_{g}, \boldsymbol{E}_{e d}=\left(\int_{0}^{\Delta t} e^{\boldsymbol{A} \tau} d \tau\right) \boldsymbol{E}_{e} . \mathrm{The}$ backward difference of the state vector, $\Delta x(k+1)=x(k+1)-x(k)$, is expressed as

$$
\Delta \boldsymbol{x}(k+1)=\boldsymbol{A}_{d} \Delta \boldsymbol{x}(k)+\boldsymbol{B}_{d} \Delta \boldsymbol{\phi}(k) .
$$

The error signal is defined as

$$
\boldsymbol{e}(k)=\boldsymbol{R}_{r}(k)-\boldsymbol{y}(k),
$$

where $\boldsymbol{R}_{r}(k)=\left[z_{r}(k) \theta_{x r}(k) \theta_{y r}(k)\right]^{T}$ represents the reference signal for vertical displacement and inclination angle of the table. From Eqs. (10) and (11), the extended state equation is obtained as

$$
\left[\begin{array}{c}
\boldsymbol{e}(k+1) \\
\Delta \boldsymbol{x}(k+1)
\end{array}\right]=\left[\begin{array}{cc}
\mathbf{I} & -\boldsymbol{C}_{d} \boldsymbol{A}_{d} \\
\mathbf{0} & \boldsymbol{A}_{d}
\end{array}\right]\left[\begin{array}{c}
\boldsymbol{e}(k) \\
\Delta \boldsymbol{x}(k)
\end{array}\right]+\left[\begin{array}{c}
-\boldsymbol{C}_{d} \boldsymbol{B}_{d} \\
\boldsymbol{B}_{d}
\end{array}\right] \Delta \boldsymbol{\phi}(k)+\left[\begin{array}{l}
\mathbf{I} \\
\mathbf{0}
\end{array}\right] \Delta \boldsymbol{R}_{r}(k+1) .
$$

When the reference signal $\boldsymbol{R}_{r}(k)$ does not change, $\Delta \boldsymbol{R}_{r}(k+1)=\mathbf{0}$ is satisfied. Therefore,

$$
\boldsymbol{X}_{0}(k+1)=\boldsymbol{\Phi} \boldsymbol{X}_{0}(k)+\boldsymbol{\Gamma} \Delta \boldsymbol{\phi}(k)
$$


is obtained, where $\boldsymbol{X}_{0}=\left[\boldsymbol{e}^{T}(k) \Delta \boldsymbol{x}^{T}(k)\right]^{T}$. The type 1 optimal servo controller can be realized by the following input

$$
\Delta \phi(k)=\left[\begin{array}{ll}
\boldsymbol{K}_{e} & \boldsymbol{K}_{x}
\end{array}\right] \boldsymbol{X}_{0}(k)=\boldsymbol{K} \boldsymbol{X}_{0}(k),
$$

where $\boldsymbol{K}_{e}$ and $\boldsymbol{K}_{x}$ are optimal feedback gain, which minimizes the evaluation function

$$
J=\frac{1}{2} \sum_{k=1}^{\infty}\left[\boldsymbol{X}_{0}^{T}(k) \boldsymbol{Q} \boldsymbol{X}_{0}(k)+\Delta \boldsymbol{\phi}^{T}(k) \boldsymbol{R} \Delta \boldsymbol{\phi}(k)\right],
$$

determined from optimal regulator theory. Here, $\boldsymbol{Q}$ and $\boldsymbol{R}$ are positive-definite weighting matrices.

\section{Identification of actuator position and effective pressure areas}

\subsection{Identification of actuator position}

This study develops an identification method of the inserted position of an additional actuator. The additional actuator, which is not included initially in the closed-loop servo-system, is inserted at the position indicated in Fig. 1. Assuming that the external force $\boldsymbol{F}_{e}$ is generated by the added actuator inserted at position $\left(x_{N+1}, y_{N+1}\right), \boldsymbol{F}_{e}$ is expressed as

$$
\boldsymbol{F}_{e}=\boldsymbol{G}_{\text {add }}\left(x_{N+1}, y_{N+1}\right) f_{N+1},
$$

where

$$
\boldsymbol{G}_{\text {add }}\left(x_{N+1}, y_{N+1}\right)=\left[\begin{array}{lll}
1 & y_{N+1} & -x_{N+1}
\end{array}\right]^{T}, f_{N+1}=A_{N+1}\left(p_{N+1}-p_{s}\right) .
$$

When displacement $\boldsymbol{X}_{0}$ is kept in state $\boldsymbol{X}_{0}=\mathbf{0}$ by using the output pressures of the actuators $\boldsymbol{p}$ excepting the (N+1)-th actuator and applying the servo controller introduced in previous section, Eq. (1) is rewritten as

$$
\boldsymbol{G}_{1} \boldsymbol{A}_{a}\left(\boldsymbol{p}-\boldsymbol{p}_{s}\right)-\boldsymbol{M g}+\boldsymbol{G}_{\mathrm{add}}\left(x_{N+1}, y_{N+1}\right) f_{N+1}=\mathbf{0}
$$

for the equilibrium state. The identification method of the inserted position of the additional actuator is expressed as the problem in solving the matrix $\boldsymbol{G}_{\text {add }}\left(x_{N+1}, y_{N+1}\right)$ of Eq. (17) by applying the force $f_{N+1}$, which is generated by the inserted actuator as

$$
\boldsymbol{G}_{\mathrm{add}}\left(\hat{x}_{N+1}, \hat{y}_{N+1}\right)=\left[\begin{array}{c}
1 \\
\hat{y}_{N+1} \\
-\hat{x}_{N+1}
\end{array}\right]=\frac{\boldsymbol{M g}-\boldsymbol{G}_{1} \boldsymbol{A}_{a}\left(\boldsymbol{p}-\boldsymbol{p}_{s}\right)}{f_{N+1}}=\frac{\boldsymbol{M g}-\boldsymbol{G}_{1} \boldsymbol{A}_{a}\left(\boldsymbol{p}-\boldsymbol{p}_{s}\right)}{A_{a(N+1)}\left(p_{N+1}-p_{s}\right)},
$$

where the hat $(\hat{o})$ symbol indicates the identified or estimated value. However, when the effective pressure areas, $A_{a i}$ $(i=1, \cdots, N+1)$, are difficult to measure directly, estimation is necessary to identify the position of the inserted actuator.

\subsection{Identification of effective pressure areas}

From the first row of the vectors in Eq. (17), the equilibrium condition of the force along the $z$-axis is given as

$$
\left(\boldsymbol{p}-\boldsymbol{p}_{s}\right)^{T}\left[\begin{array}{lll}
A_{a 1} & \cdots & A_{a N}
\end{array}\right]^{T}+\left(p_{N+1}-p_{s}\right) A_{a(N+1)}=\left(\left[p_{1} \cdots p_{N} \mid p_{N+1}\right]-\left(\boldsymbol{p}_{s(N \times 1)}\right)^{T}\right) \boldsymbol{A}_{a}^{*}=M g .
$$

Given more than $(N+1)$ different equilibrium conditions achieved by changing the pressure, $p_{N+1}$, generated by the inserted actuator, we can construct a matrix equation

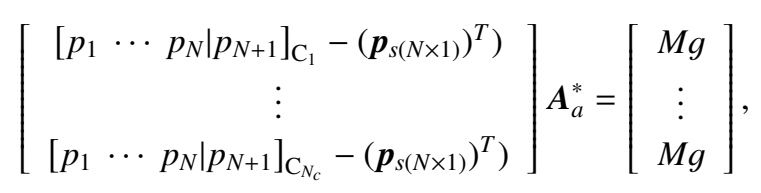

where $\mathrm{C}_{j}$ and $N_{c}\left(N_{c} \geq N+1\right)$ represents the $j$-th equilibrium condition and the number of equilibrium conditions, respectively. Rewriting Eq. (20) as

$$
\Pi \boldsymbol{A}_{a}^{*}=\boldsymbol{\Psi},
$$

the vector $\boldsymbol{A}_{a}^{*}$ can be solved by applying the least squares method or by using the pseudo inverse matrix $\boldsymbol{\Pi}^{\dagger}$ as

$$
\boldsymbol{A}_{a}^{*}=\boldsymbol{\Pi}^{\dagger} \boldsymbol{\Psi} .
$$

Finally, we can estimate the effective pressure areas of the pneumatic actuators by Eq. (22) and the position of the inserted actuator by Eq. (18). 


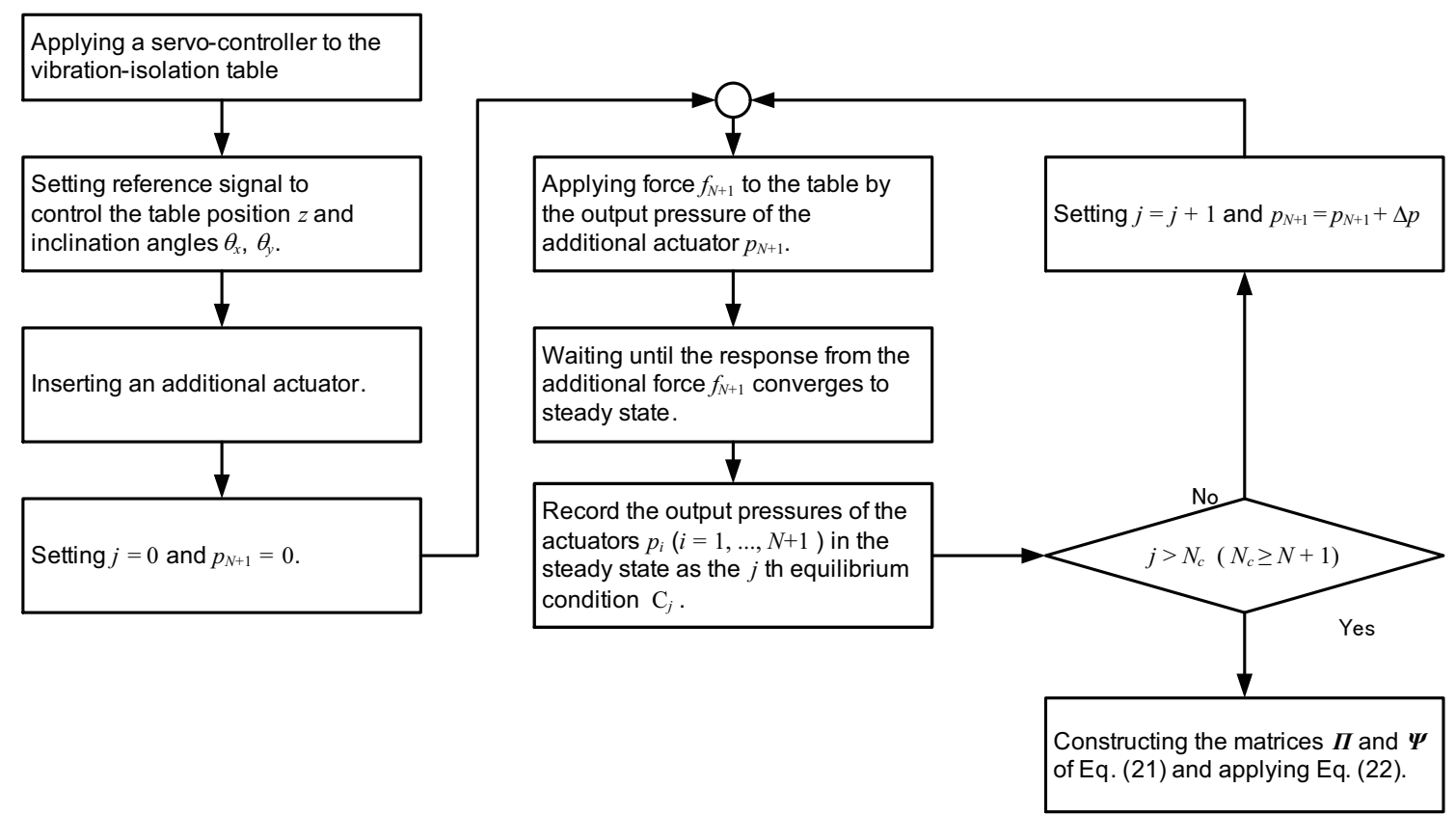

Fig. 3 Identification procedure of the effective pressure areas of pneumatic actuators.

\subsection{Identification procedure}

The identification procedure using Eq. (22) is shown in Fig. 3. Equilibrium conditions $C_{j}\left(j=1, \cdots, N_{c}\right)$ in Eq. (22), necessary for identification of effective pressure areas, are realized by using the additional actuator output. Adding an actuator at the approximately appropriate position as shown in Fig. 1 and applying force $f_{N+1}$ to the closed loop system (type 1 digital servo system given by Eqs. (10) and (14)), the closed loop system converges to an equilibrium state $\mathrm{C}_{j}$. In the equilibrium condition $\mathrm{C}_{j}$, both $\boldsymbol{e}(\boldsymbol{k}) \Rightarrow \mathbf{0}$ and Eq. (19) are satisfied under different output pressures, $p_{N+1}$. Constructing the matrices $\boldsymbol{\Pi}$ and $\boldsymbol{\Psi}$, the effective pressure areas are identified as $\boldsymbol{A}_{a}^{*}$ by Eq. (22). Further, by applying Eq. (18), the position of the added actuator $\left(x_{N+1}, y_{N+1}\right)$ is estimated.

\section{Results of Simulation}

Parameters of the vibration-isolation table are shown in Table 1. In this study, the number of actuators, $N$, are set to eight initially, and arrangement of the eight actuators at initial conditions is shown in Table 2. Setting the weighting

Table 1 Parameters of the mathematical model for vibration-isolation table.

\begin{tabular}{ccc|ccc}
\hline$M$ & $5.30 \times 10^{2}$ & $\mathrm{~kg}$ & $g$ & 9.80 & $\mathrm{~m} / \mathrm{s}^{2}$ \\
$J_{x}$ & 29.6 & $\mathrm{~kg} \cdot \mathrm{m}^{2}$ & $R$ & 287 & $\mathrm{~J} / \mathrm{kg} \cdot \mathrm{K})$ \\
$J_{y}$ & 30.1 & $\mathrm{~kg} \cdot \mathrm{m}^{2}$ & $\kappa$ & 1.40 & - \\
$T_{0}$ & 293 & $\mathrm{~K}$ & $p_{0 i}$ & 0.184 & $\mathrm{MPa}$ \\
$z_{0 i}$ & $2.00 \times 10^{-2}$ & $\mathrm{~m}$ & $K_{u}$ & $5.03 \times 10^{-5}$ & $\mathrm{rad} / \mathrm{pulse}$ \\
$p_{s}$ & 0.1013 & $\mathrm{MPa}$ & $H_{i}$ & $2.20 \times 10^{4}$ & $\mathrm{~Pa} / \mathrm{rad}$ \\
$L_{i}$ & $1.00 \times 10^{-2}$ & $\mathrm{~m}$ & $\gamma_{i}$ & 55.0 & $1 / \mathrm{s}$ \\
$A_{a i}$ & $7.85 \times 10^{-3}$ (Nominal value) & $\mathrm{m}^{2}$ & $c_{i}$ & $6.30 \times 10^{-7}$ & $\mathrm{~m} \cdot \mathrm{s}$ \\
\hline
\end{tabular}

Table 2 Positions of pneumatic actuators at the initial conditions.

\begin{tabular}{l|rrc|l|rrr}
\hline$\# 1$ & $x_{1}$ & -272 & $\mathrm{~mm}$ & $\# 5$ & $x_{5}$ & 272 & $\mathrm{~mm}$ \\
& $y_{1}$ & -272 & $\mathrm{~mm}$ & & $y_{5}$ & 272 & $\mathrm{~mm}$ \\
\hline$\# 2$ & $x_{2}$ & 0 & $\mathrm{~mm}$ & $\# 6$ & $x_{6}$ & 0 & $\mathrm{~mm}$ \\
& $y_{2}$ & -272 & $\mathrm{~mm}$ & & $y_{6}$ & 272 & $\mathrm{~mm}$ \\
\hline$\# 3$ & $x_{3}$ & 272 & $\mathrm{~mm}$ & $\# 7$ & $x_{7}$ & -272 & $\mathrm{~mm}$ \\
& $y_{3}$ & -272 & $\mathrm{~mm}$ & & $y_{7}$ & 272 & $\mathrm{~mm}$ \\
\hline$\# 4$ & $x_{4}$ & 272 & $\mathrm{~mm}$ & $\# 8$ & $x_{8}$ & -272 & $\mathrm{~mm}$ \\
& $y_{4}$ & 0 & $\mathrm{~mm}$ & & $y_{8}$ & 0 & $\mathrm{~mm}$ \\
\hline
\end{tabular}


matrices $\boldsymbol{Q}$ and $\boldsymbol{R}$ in Eq. (15) to

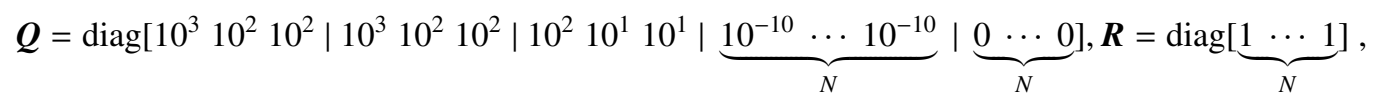

the feedback gain of a type 1 digital servo system, $\boldsymbol{K}$, is determined. First, the vibration-isolation table is supported by the eight pneumatic actuators, and the position and inclination angles are set to and kept in $z=0 \mathrm{~mm}, \theta_{x}=0 \mathrm{rad}$, and $\theta_{y}=0 \mathrm{rad}$ by the servo controller. Next, we insert a pneumatic actuator at the position $\left(x_{N+1}, y_{N+1}\right)=(0.136 \mathrm{~mm}, 0,136$ $\mathrm{mm})$. It is assumed that the added actuator position is only approximately known, however, the position $\left(x_{N+1}, y_{N+1}\right)$ can be estimated by using the proposed method as shown in the following subsection.

\subsection{Identification results}

First, the effective pressure areas are identified by the procedure shown in Fig. 3. The initial output force of the added actuator, $f_{N+1}$, is increased by $2 \mathrm{~N}$ steps by a step function every $3 \mathrm{~s}$ from $0 \mathrm{~N}$ to $1600 \mathrm{~N}\left(N_{c}=800\right)$. The steady-state pressures of all actuators are obtained to construct the matrix $\boldsymbol{\Pi}$ in Eq. (21) in each step. The identified effective pressure areas are obtained by Eq. (22) and the identification results of the effective pressure areas are shown in Table 3. Here, actual values with uncertainty are assumed as unknown values and identified by the proposed method. The actual and identified values are shown in the upper and lower lines of Table 3, respectively. Here, relative identification errors of the effective pressure areas are calculated by

$$
\frac{\hat{A}_{a i}-A_{a i}}{A_{a i}} \times 100[\%]
$$

Identification results which have less than approximately $8 \%$ of the relative identification errors are obtained.

Next, using the estimated results of the effective pressure areas, the position of the added actuator is also estimated by Eq. (18). Identification result of the position of the added actuator is shown in Table 4. Actual values assumed as unknowns and the identified values are shown in the left and right columns of Table 4, respectively. The obtained results were achieved to a very high degree of accuracy. Here, the relative estimation errors calculated by

$$
\frac{\hat{x}_{9}-x_{9}}{x_{9}} \times 100[\%], \frac{\hat{y}_{9}-y_{9}}{y_{9}} \times 100[\%]
$$

are indicated in the parentheses in Table 4.

\subsection{Application example}

As an example of an application of the proposed identification method, this section demonstrates the method for increasing the number of actuators of the vibration-isolation table (supported by a redundant number of actuators) while working continuously, and shows the method for obtaining adaptability to the desired load specification. Note that it is difficult to construct the method for decreasing the number of actuators because the method has to realize removing the

\begin{tabular}{|c|c|c|c|c|c|c|c|}
\hline \multirow{3}{*}{$\begin{array}{c}\text { No. } \\
\# 1\end{array}$} & $\overline{\text { Effe }}$ & re areas $\left[\mathrm{m}^{2}\right]$ & Relative error [\%] & No. & $\overline{\text { Effe }}$ & re areas $\left[\mathrm{m}^{2}\right]$ & Relative error [\%] \\
\hline & $A_{a 1}$ & $7.46 \times 10^{-3}$ & \multirow[b]{2}{*}{5.28} & \multirow[t]{2}{*}{$\# 5$} & $A_{a 5}$ & $8.01 \times 10^{-3}$ & \multirow[b]{2}{*}{0.98} \\
\hline & $\hat{A}_{a 1}$ & $7.86 \times 10^{-3}$ & & & $\hat{A}_{a 5}$ & $8.08 \times 10^{-3}$ & \\
\hline \multirow[t]{2}{*}{$\# 2$} & $A_{a 2}$ & $8.25 \times 10^{-3}$ & \multirow[b]{2}{*}{2.68} & \multirow[t]{2}{*}{ \#6 } & $A_{a 6}$ & $8.64 \times 10^{-3}$ & \multirow[b]{2}{*}{-0.37} \\
\hline & $\hat{A}_{a 2}$ & $8.47 \times 10^{-3}$ & & & $\hat{A}_{a 6}$ & $8.60 \times 10^{-3}$ & \\
\hline \multirow[t]{2}{*}{ \#3 } & $A_{a 3}$ & $7.62 \times 10^{-3}$ & \multirow[b]{2}{*}{2.02} & \multirow[t]{2}{*}{$\# 7$} & $A_{a 7}$ & $7.78 \times 10^{-3}$ & \multirow[b]{2}{*}{-8.07} \\
\hline & $\hat{A}_{a 3}$ & $7.77 \times 10^{-3}$ & & & $\hat{A}_{a 7}$ & $7.15 \times 10^{-3}$ & \\
\hline \multirow[t]{4}{*}{ \#4 } & $A_{a 4}$ & $7.23 \times 10^{-3}$ & \multirow[b]{2}{*}{-5.27} & \multirow[t]{2}{*}{$\# 8$} & $A_{a 8}$ & $8.17 \times 10^{-3}$ & \multirow[b]{2}{*}{0.45} \\
\hline & $\hat{A}_{a 4}$ & $6.84 \times 10^{-3}$ & & & $\hat{A}_{a 8}$ & $8.21 \times 10^{-3}$ & \\
\hline & & & & $\# 9$ & $\overline{A_{a 9}}$ & $7.85 \times 10^{-3}$ & \multirow[b]{2}{*}{1.38} \\
\hline & & & & (Added) & $\hat{A}_{a 9}$ & $7.75 \times 10^{-3}$ & \\
\hline
\end{tabular}
active and working actuator smoothly and changing the controller continuously, so the authors are currently investigating

Table 3 Identification results of the effective pressure areas.

Table 4 Identification results of the position of the added actuator.

\begin{tabular}{c|ccc|c|cccc}
\hline$\# 9$ & $x_{9}$ & 136 & $\mathrm{~mm}$ & $\# 9$ & $\hat{x}_{9}$ & 136.0059 & $\mathrm{~mm}$ & $(0.0043 \%)$ \\
(added) & $y_{9}$ & 136 & $\mathrm{~mm}$ & (Estimated) & $\hat{y}_{9}$ & 136.0048 & $\mathrm{~mm}$ & $(0.0035 \%)$ \\
\hline
\end{tabular}




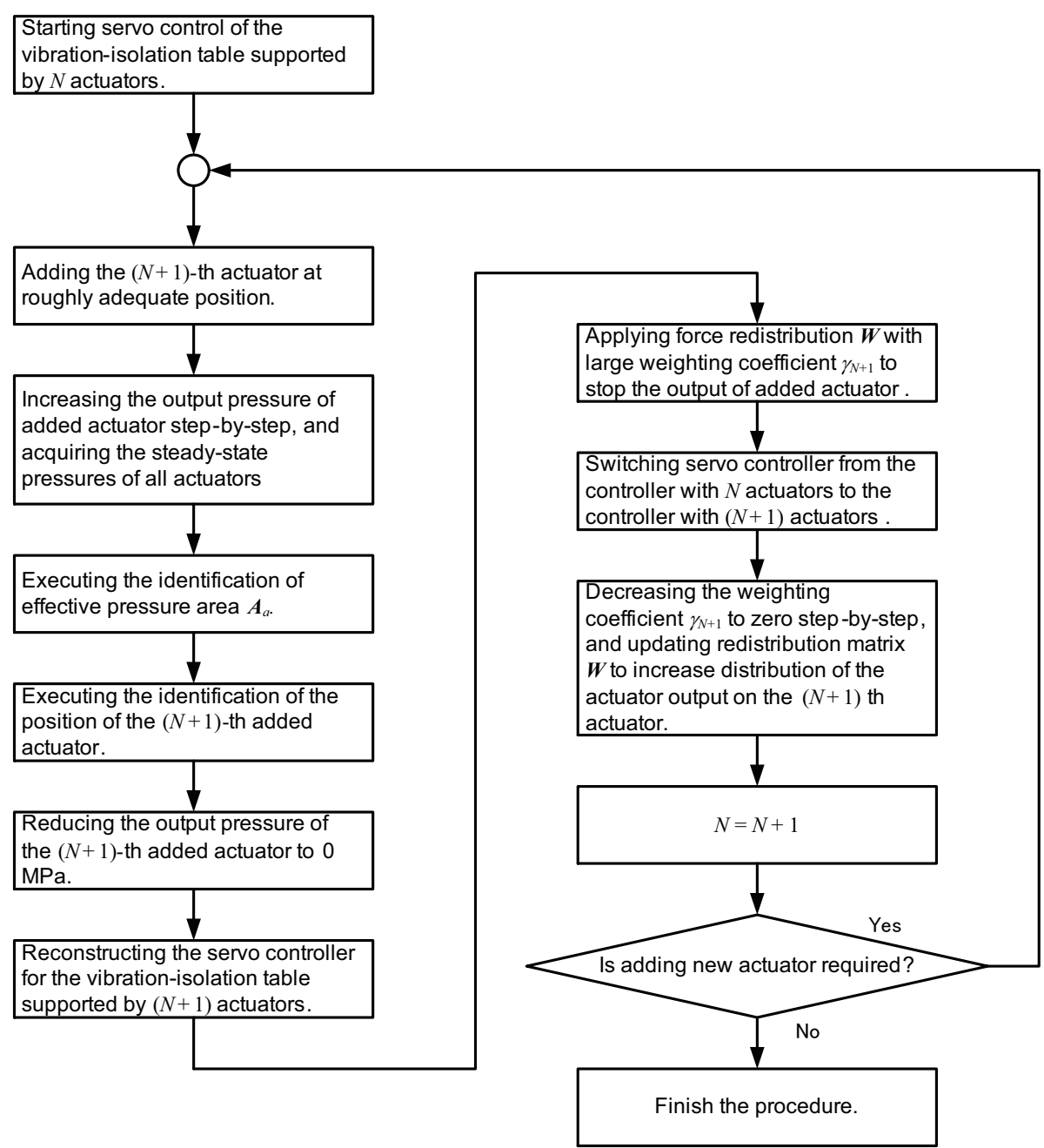

Fig. 4 Procedure for increasing the number of actuators which support a vibration-isolation table.

the method for decreasing the number of actuators smoothly. The procedure is outlined in Fig. 4. First, applying the identification method of the effective pressure areas given by Eq. (22), the effective pressure areas $\boldsymbol{A}_{a}$ are estimated and the position of the $(N+1)$-th added actuator is estimated by Eq. (18). The servo controller $\boldsymbol{K}$ for the vibration-isolation table supported by $(N+1)$ actuators is prepared. Applying force redistribution (Hoshino et al., 2012, Maeda et al., 2013) represented by a redistribution matrix $\boldsymbol{W}(k)$ as

$$
\boldsymbol{A}_{a} \boldsymbol{K}_{1} \boldsymbol{\phi}^{\prime}(k)=\boldsymbol{W}(k) \boldsymbol{G}_{1} \boldsymbol{A}_{a} \boldsymbol{K}_{1} \boldsymbol{\phi}(k),
$$

the $(N+1)$-th actuator is introduced into the servo controller (which means the $(N+1)$-th added actuator is activated as one of the actuators of the servo controller). Here, the forces generated by each actuator to yield $\boldsymbol{F}^{*}=\left[F^{*}, M_{x}^{*}, M_{y}^{*}\right]^{T}$ are obtained by the least-squares method (Hoshino et al., 2012, Maeda et al., 2013). For adequate distribution of output force on the $i$-th actuator, a cost function is defined as

$$
S=\frac{1}{2} \alpha_{F}\left(F-F^{*}\right)^{2}+\frac{1}{2} \alpha_{x}\left(M_{x}-M_{x}^{*}\right)^{2}+\frac{1}{2} \alpha_{y}\left(M_{y}-M_{y}^{*}\right)^{2}+\frac{1}{2} \alpha_{\Delta} \sum_{i=1}^{N+1}\left(f_{i}-\frac{F}{N}\right)^{2}+\frac{1}{2} \sum_{i=1}^{N+1} \gamma_{i} f_{i}^{2},
$$

where $\alpha_{F}, \alpha_{x}, \alpha_{y}, \alpha_{\Delta}$, and $\gamma_{i}$ represent non-negative weighting coefficients. Additional details are given in the references (Hoshino et al., 2007, 2010, 2012, Maeda et al., 2013). The fifth term is the sum of the squares of the forces generated by the actuators and is introduced to consider the conditions where the force distribution on the $i$-th actuator is stopped and, consequently, the force is appropriately or optimally redistributed onto the other actuators. The force vector $\boldsymbol{f}=\boldsymbol{A}_{a}\left(\boldsymbol{p}-\boldsymbol{p}_{s}\right)$ that minimizes the cost function (27) can be expressed as

$$
\boldsymbol{f}=\boldsymbol{W} \boldsymbol{F}^{*}
$$

where $\boldsymbol{W}$ represents the transformation matrix obtained by the least-squares method that minimizes the cost function (27). Setting $\gamma_{i}$ to a large value, the pressure distributed on the $i$-th actuator is stopped (Hoshino et al., 2012, Maeda et al., 
2013). Setting $\gamma_{N+1}(k)$ to 1000 , which is sufficiently large value to stop the distribution (Hoshino et al., 2012, Maeda et al., 2013), $\boldsymbol{W}(k)$ is derived to stop the distribution on the $(N+1)$-th actuator. Then the controller is switched from the controller for the vibration-isolation table supported by $N$ actuators to one which uses $(N+1)$ actuators. In this study, the type 1 digital servo controller is determined by substituting $(N+1)$ into $N$ in Eq. (23). Decreasing $\gamma_{N+1}(k)$ step-by-step to 0 and updating $\boldsymbol{W}(k)$ at the each sample step of the controller, the $(N+1)$-th actuator is activated gradually and the distributed force on the $(N+1)$-th actuator increases gradually as well. Although $\boldsymbol{W}$ has been derived by assuming the weighting coefficient $\gamma_{N+1}$ with a constant value stated in the reference (Hoshino et al., 2012, Maeda et al., 2013), this paper extends the method for the case when $\gamma_{N+1}$ is changed step-by-step at each sample step of the controller.

From Eq. (26), force $\boldsymbol{f}^{\prime}(k)$ obtained by $\boldsymbol{W}(k)$ at the sample step $k$ and $(k-1)$ are expressed as

$$
\boldsymbol{f}^{\prime}(k)\left(=\boldsymbol{A}_{a}\left(\boldsymbol{p}^{\prime}(k)-\boldsymbol{p}_{s}\right)\right)=\boldsymbol{A}_{a} \boldsymbol{K}_{1}\left(\boldsymbol{\phi}^{\prime}(k)-\boldsymbol{\phi}_{0}\right)=\boldsymbol{W}(k) \boldsymbol{G}_{1} \boldsymbol{A}_{a} \boldsymbol{K}_{1}\left\{\boldsymbol{\phi}(0)+\sum_{i=1}^{k} \Delta \boldsymbol{\phi}(i)-\boldsymbol{\phi}_{0}\right\},
$$

and

$$
\boldsymbol{f}^{\prime}(k-1)\left(=\boldsymbol{A}_{a}\left(\boldsymbol{p}^{\prime}(k-1)-\boldsymbol{p}_{s}\right)\right)=\boldsymbol{A}_{a} \boldsymbol{K}_{1}\left(\boldsymbol{\phi}^{\prime}(k-1)-\boldsymbol{\phi}_{0}\right)=\boldsymbol{W}(k-1) \boldsymbol{G}_{1} \boldsymbol{A}_{a} \boldsymbol{K}_{1}\left\{\boldsymbol{\phi}(0)+\sum_{i=1}^{k-1} \Delta \boldsymbol{\phi}(i)-\boldsymbol{\phi}_{0}\right\},
$$

where $\boldsymbol{p}_{s}=\boldsymbol{K}_{1} \boldsymbol{\phi}_{0}$. Subtracting Eq. (30) from (29), the backward difference of the redistributed force is derived as

$$
\Delta \boldsymbol{f}^{\prime}(k)=\boldsymbol{A}_{a} \boldsymbol{K}_{1} \Delta \boldsymbol{\phi}^{\prime}(k)=\{\boldsymbol{W}(k)-\boldsymbol{W}(k-1)\} \boldsymbol{G}_{1} \boldsymbol{A}_{a} \boldsymbol{K}_{1}\left\{\boldsymbol{\phi}(0)+\sum_{i=1}^{k-1} \Delta \boldsymbol{\phi}(i)-\boldsymbol{\phi}_{0}\right\}+\boldsymbol{W}(k) \boldsymbol{G}_{1} \boldsymbol{A}_{a} \boldsymbol{K}_{1} \Delta \boldsymbol{\phi}(k) .
$$

Substituting

$$
\phi(k)=\phi(0)+\sum_{i=1}^{k} \Delta \phi(i)
$$

into Eq. (31), the dynamically redistributed control input $\Delta \boldsymbol{\phi}^{\prime}(k)$ obtained from $\Delta \phi(k)$ is derived as

$$
\Delta \boldsymbol{\phi}^{\prime}(k)=\boldsymbol{K}_{1}^{-1} \boldsymbol{A}_{a}^{-1}\left[\boldsymbol{W}(k) \boldsymbol{G}_{1} \boldsymbol{A}_{a} \boldsymbol{K}_{1} \Delta \boldsymbol{\phi}(k)+\{\boldsymbol{W}(k)-\boldsymbol{W}(k-1)\} \boldsymbol{G}_{1} \boldsymbol{A}_{a} \boldsymbol{K}_{1}\left\{\boldsymbol{\phi}(k-1)-\boldsymbol{\phi}_{0}\right\}\right] .
$$

Note that the aforementioned procedure, as shown in Fig. 4, can be applied recursively to accommodate any increase in the number of actuators.

Simulation results are shown in Fig. 5. In this simulation, the vibration-isolation table is supported by eight pneumatic actuators with position $z$ and inclination angles $\left(\theta_{x}, \theta_{y}\right)$ controlled by the type 1 digital servo system designed for the model
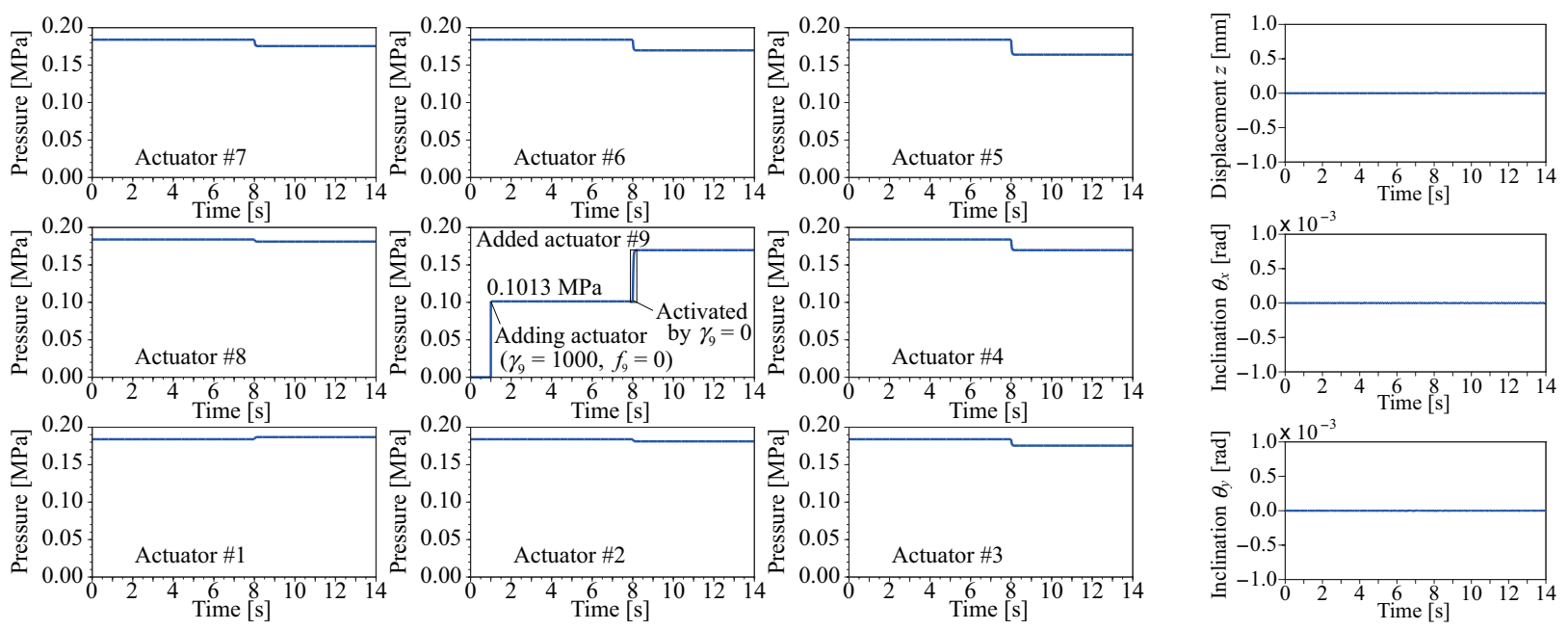

(a) Pressures of actuators

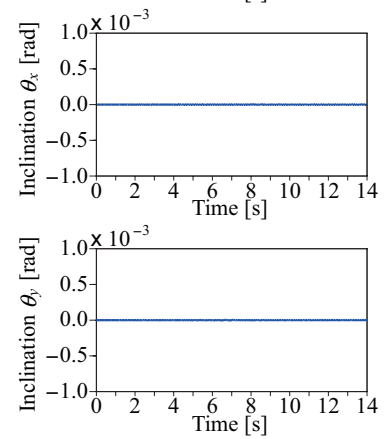

(b) Displacement $z$ and inclination angles $\left(\theta_{x}, \theta_{y}\right)$

Fig. 5 Time histories of the pressures of actuators and the displacement $z$ and the inclination angles $\left(\theta_{x}, \theta_{y}\right)$ of table when increasing the number of actuators from 8 to 9 by using the proposed procedure. In this simulation, the vibration-isolation table is supported by eight actuators and position $z$ and inclination angles $\left(\theta_{x}, \theta_{y}\right)$ are controlled by the type 1 digital servo system designed for the model with eight actuators, and then actuator \#9 is inserted. By first setting $\gamma_{9}$ to 1000 to stop distribution of the force on the actuator \#9, the controller is switched to the model with nine actuators. Then, decreasing $\gamma_{9}$ gradually, the distribution of the force on the actuator \#9 is increasing after $8 \mathrm{~s}$. 


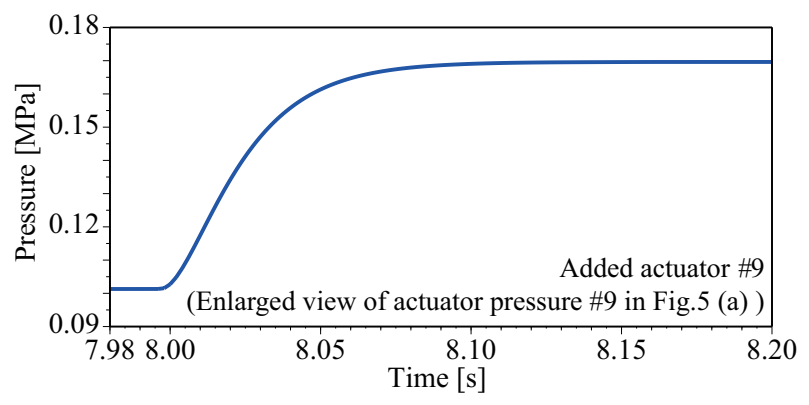

Fig. 6 Enlarged view of the actuator pressure \#9. This figure shows enlarged view of the region around $8 \mathrm{~s}$ indicated by the square in the figure of actuator pressure \#9 of Fig. 5 (a) and shows that smooth change in distribution rate is achieved.

of table supported by eight actuators. Then, actuator \#9 was placed at position $\left(x_{9}, y_{9}\right)=(136 \mathrm{~mm}, 136 \mathrm{~mm})$. By first setting $\gamma_{9}$ to 1000 to stop distribution of the force on the actuator \#9, which then redistributes the force optimally to the other actuators by the proposed redistribution method in Eq. (32), the controller is switched to the model which contains nine actuators. Then, decreasing $\gamma_{9}$ gradually, the distribution of the force on the actuator \#9 is increasing from around $8 \mathrm{~s}$, while the actuator forces generated by all actuators (including the actuator \#9) are maintaining appropriate force distribution by the redistribution method Eq. (32). Figure 6 shows enlarged view of the region indicated by the square in the figure of actuator pressure \#9 of Fig. 5 (a). We can see smooth change in distribution rate from Fig. 6. Finally, the displacement and inclination angles are controlled by using all nine actuators. Through this simulation, smooth responses in the displacement and inclination angles were observed despite switching both the controller and the number of actuators (Fig. 5 (b)).

\section{Conclusions}

This study investigated a self-identification method of positions and effective pressure areas of pneumatic actuators which support a vibration-isolation table containing a redundant number of actuators. The proposed identification method can be represented simply by a self-identification algorithm. This study evaluated the accuracy of the estimation values and reliability of the estimated parameters for constructing the controller such as linear quadratic regulators (LQR) to configure the control system automatically. By using the proposed method, we demonstrated the simple addition of an actuator into the vibration-isolation system according to the load change. In addition, by using this process recursively, the method can be extended to accommodate the desired number of actuators into the active control system of the vibrationisolation table.

\section{Acknowledgments}

This work was supported by JSPS KAKENHI (Multi-year Fund) Grant Number (25870010).

\section{References}

Chen, P.-C. and Shih, M.-C., Robust Control of a Novel Active Pneumatic Vibration Isolator through Floor Vibration Observer, Journal of Vibration and Control, Vol. 17, No. 9 (2010), pp. 1325-1336.

Erin, C., Wilson, B. and Zapfe, J., An Improved Model of a Pneumatic Vibration Isolator: Theory and Experiment, Journal of Sound and Vibration, Vol. 218, No. 1 (1998), pp. 81-101.

Hashimoto, S., Mizutani, K. and Ikeura, R., Design of a Control System for Three-Degree-of-Freedom Hybrid Vibration Isolation System, Proceedings of Dynamics and Design Conference 2007 (D\&D2007) (2007), 414 (in Japanese).

Hoshino, Y., Kobayashi, Y., Chinda, H. and Nakanishi, M., Active Vibration and Attitude Control of a Vibration Isolation Table with Pneumatic Actuators (A Modeling Method for the Redundant System of Actuators and Sensors), Transactions of the Japan Society of Mechanical Engineers Series C, Vol. 73, No. 727 (2007), pp. 677-684 (in Japanese).

Hoshino, Y., Kobayashi, Y., Chinda, H. and Takahashi, M., Minimum-Order System Identification by Subspace Method and Frequency-Domain Least-Squares Approximation for Vibration Isolation Tables, Proceedings of 2010 ASME International Mechanical Engineering Congress and Exposition (IMECE2010) (2010), Paper No. IMECE2010-40250. 
Hoshino, Y., Kobayashi, Y., Morita, T., Chinda, H. and Takahashi, M., System Identification of Minimum-Order-Model for Vibration Isolation Table by Subspace Method and Frequency-Domain Least-Squares Approximation, Transactions of the Japan Society of Mechanical Engineers Series C, Vol. 75, No. 749 (2009), pp. $42-49$ (in Japanese).

Hoshino, Y., Maeda, T., Yoshida, D., Kobayashi, Y. and Emaru, T., A Vibration Isolation Table Supported by a Redundant Number of Pneumatic Actuators and Force Redistribution for Compensating Broken Actuators, Proceedings of 2012 IEEE/SICE International Symposium on System Integration (SII2012) (2012), pp. 847-852, Paper No. MP2-A.3.

Hoshino, Y., Yoshida, D., Maeda, T., Kobayashi, Y. and Emaru, T., Competitive Pressure Modes in a Vibration-Isolation Table Supported by a Redundant Number of Pneumatic Actuators and its Stabilization Method, Proceedings of the 15th Asia Pacific Vibration Conference (APVC2013) (2013), pp. 1372-1377.

Kim, S.-M., Elliott, S. J. and Brennan, M. J., Decentralized Control for Multichannel Active Vibration Isolation, IEEE Transactions on Control Systems Technology, Vol. 9, No. 1 (2001), pp. 93-100.

Maeda, T., Hoshino, Y., Yoshida, D, Kobayashi, Y. and Emaru, T., Force Redistribution Method for Compensating Actuator-Breakdown of Vibration-Isolation Tables Supported with a Redundant Number of Pneumatic Actuators, Journal of System Design and Dynamics, Vol. 7, No. 4 (2013), pp. 355-366.

Shin, Y.-H., Kim, K.-J., Chang, P.-H. and Han, D.-K., Three Degrees of Freedom Active Control of Pneumatic Vibration Isolation Table by Pneumatic and Time Delay Control Technique, Transactions of ASME, Journal of Vibration and Acoustics, Vol. 132, No. 5 (2010), pp. 1-12.

Shirani, H. and Wakui, S., Feedforward Control of the Flow Disturbance to the Pneumatic Isolation Table, Journal of System Design and Dynamics, Vol. 4, No. 5 (2010), pp. 672-682.

Zuo, L., Slotine, J. J. E. and Nayfeh, S. A., Model Reaching Adaptive Control for Vibration Isolation, IEEE Transactions on Control Systems Technology, Vol. 13, No. 4 (2005), pp. 611-617. 